\title{
Exploring what constitutes effective continued healthcare professional training: A qualitative study and the development of a training intervention component taxonomy.
}

Katherine Perryman ( $\sim$ katherine.perryman@manchester.ac.uk )

The University of Manchester Faculty of Biology Medicine and Health https://orcid.org/0000-0003-

1275-6991

Lis Cordingley

University of Manchester

Christine Bundy

Cardiff University

Research article

Keywords: Healthcare professional training, continued education, taxonomy, quality improvement, behaviour change

Posted Date: April 1st, 2020

DOI: https://doi.org/10.21203/rs.3.rs-19808/v1

License: (c) (i) This work is licensed under a Creative Commons Attribution 4.0 International License.

Read Full License 


\section{Abstract}

Background Continued healthcare professional training can lead to improvements in patient care if delivered effectively but there is a lack of research exploring the key factors that underpin successful training. The aims of this research were to first identify what healthcare professionals and experts in training/behaviour change perceive to be effective training, second to produce a framework for delivering training optimised for effectiveness, and third create a taxonomy of training intervention components to be used for designing, reporting and evaluating healthcare professional training.

Methods Two semi-structured qualitative interview studies were conducted: Study 1 participants were 18 healthcare professionals who had attended training to implement collaborative care for the management of depression and anxiety in patients with long term conditions; Study 2 participants were 16 experts in training or behaviour change. Data were analysed using framework analyses. A preliminary framework of what constitutes effective training was developed from Study 1 data, then tested and adapted using Study 2 data. Intervention components identified as effective by participants in both studies were defined and grouped to form a taxonomy of training intervention components. They were mapped onto 38 intervention descriptions to assess breadth of coverage.

Results Three core concepts (interpersonal, intrapersonal and system factors) and five themes (social interaction, credibility, relevance, information processing, practicalities) that captured what participants perceived to underpin effective training were identified. These were organised into the Perceived Effectiveness of Training framework. A list of training components linked to each theme was developed from the data and a taxonomy of 171 defined training intervention components organized into three phases and grouped into clusters was developed.

Conclusions This research identified key attributes that constitute effective training as perceived by a sample of healthcare professionals and experts in training/behaviour change. The framework can be used to design and deliver training that is optimised for effectiveness, or as a basis for training evaluation. The taxonomy can be used as a resource to identify effective training components and offers a common language to describe training interventions. Future research may be useful to further explore in what contexts these components are most effective.

\section{Background}

The rise in the availability of evidence-based treatments and clinical guidelines has been accompanied by workplace training programmes that aim to change healthcare professional behaviour so that the most up to date, evidenced based healthcare is delivered to patients. Healthcare professional training interventions are common quality improvement strategies to reduce ineffective or suboptimal care and can be effective in changing the practice of healthcare professionals (1). Training is one of the strategies identified in the Behaviour Change Wheel, a framework for designing and characterising behaviour change interventions (2). Training interventions primarily involve preparing someone to perform a task or 
a role and may involve the acquisition of new behaviours or attempts to change existing behaviour. It is often used in health services research as a component of a trial or study that aims to test the efficacy or implementation of an intervention delivered by healthcare professionals. Effective continued healthcare professional training is a crucial stage in the implementation of evidence based practice.

Training that is well designed and based on scientific theory often yields positive results (3). However, there is a lack of agreement on what constitutes effective healthcare professional training to achieve the best results. In the wider field of continued medical education (CME; which includes healthcare professional training interventions) it is difficult to determine effective components of interventions due to a lack of theory underpinning delivery and evaluation, as well as poor quality intervention descriptions in the literature (4-6). A scientifically sound body of knowledge of what constitutes effective training interventions could improve the quality of training delivered to healthcare professionals, improve clinical practice and reduce the delivery of costly ineffective training. However, poor intervention descriptions undermine replication of studies and hinder the identification of effective interventions.

The CONSORT statement for non-pharmacological randomized controlled trials recommends that details of the delivery of interventions and descriptions of intervention components are reported (7). However, there is no guidance on what these descriptions should consist of and the development of standardized terminology for the specification of interventions is needed (8). To our knowledge, there is no existing taxonomy of healthcare professional training intervention components. Existing taxonomies or guidance that aims to improve the reporting of education or behaviour change interventions (8-12) do not specify intervention components commonly used in training interventions (e.g. lectures, interactive presentations, role play, group discussion). The development of a taxonomy of training intervention components to improve the design, reporting and evaluation of healthcare professional training could therefore be of value in the quest to improve scientific knowledge of effective healthcare professional training.

Furthermore, there are a number of theories that can be used to design healthcare training and evaluate its effectiveness. For example, Social Cognitive Theory (13-15) is relevant as both a learning theory and a behaviour change theory. Explicit use of theories in a training context may have more impact on healthcare professionals by identifying specific mechanisms by which to target improvements in learners' knowledge, skills, attitudes, confidence/self-efficacy, motivation or behaviour in practice. Ineffective training wastes time and resources and there is onus on training providers to ensure quality and to maximize opportunities for impact.

Qualitative investigative approaches based on in-depth exploration and engagement with participants' experiences are particularly suitable for investigating what constitutes effective healthcare professional training. Analysis and interpretation of qualitative research data in the context of explicit educational or psychological theory enables subjective perceptions of effective training to be linked with putative mechanisms of action. This approach can uncover why certain training components are perceived as effective, enabling specific recommendations to be made (16). 
This paper reports two qualitative studies conducted with healthcare professionals and established experts in training and behaviour change. The studies aimed to explore the perceived effectiveness of healthcare professional training methods and associated theoretical constructs. A theoretically informed, evidence based, hierarchical taxonomy of training intervention components was developed which can be used to improve the design, reporting and evaluation of healthcare professional training.

\section{Methods}

\section{Design}

Two sequential qualitative studies were undertaken with two different target groups of participants to investigate what constitutes effective healthcare professional training. The first group were the recipients of continued healthcare professional training and the second were experts in training and/or behavioural change. The framework method $(17,18)$ guided the design and analyses of the study.

\section{Participants}

Study 1

Semi-structured individual telephone interviews were conducted in 2011 with 18 healthcare professionals. An opportunistic sampling strategy was adopted for this study, which was conducted as part a pilot study to inform an associated randomised controlled trial of collaborative care for depression in patients with diabetes and/or coronary heart disease (19). The pilot study evaluated a five-day training programme that included a variety of training methods (didactic and interactive presentations; demonstration role play; role play/skills practice). The training was conducted in a group workshop setting, facilitated by clinicians and academic experts with relevant experience. It was delivered to Psychological Wellbeing Practitioners (PWPs) who were acting as case managers with a remit to provide brief psychological interventions for depression and anxiety in patients with long term conditions. There was also a one day training event using similar methods attended by primary care practice nurses (PNs) and the PWPs they would be collaborating with in practice.

The researcher $(\mathrm{KP})$ observed the training and distributed participant information sheets and consent forms to the trainees. All 33 attendees (16 PWPs and 17 Practice Nurses) were contacted by email in the first instance to request participation in the study. Those who did not respond by email were contacted by telephone and interested participants were asked to provide written consent prior to interview. Eighteen attendees consented to be interviewed: 10 PWPs (4 trainees, 6 qualified) and 8 practice nurses. One of the practice nurses recruited was a prescribing support pharmacist by professional background, but was taking the role of PN in the pilot study, thus is described as a PN to preserve anonymity. Also, only one participant was male, therefore all participants were referred to using female pronouns (she, her) when necessary to maintain anonymity. 
A topic based framework matrix was used to inform the topic guides for the interviews which explored perceptions of the training format (how it was delivered); content (what was delivered); training methods (e.g. group discussion, role play); characteristics (group size, setting, length) and organizational/implementation issues (barriers and facilitators to implementing the training objectives). Questions were asked about the specific methods used in the training participants had attended and also healthcare professional training in general. For example: "What did you think of the lectures presenting the background information? What do you think about lectures as a learning method? Are there any other ways you would like to receive this type of information?" Two separate topic guides (one for the PWPs and one for the PNs) were developed reflecting the content of the two training sessions. This was piloted on the first few participants and emerging issues were added to subsequent interviews. All interviews were conducted by telephone, audio recorded and transcribed verbatim by an established transcription service and quality checked for accuracy.

Study 2

A purposive sampling strategy identified 19 experts in behaviour change/training. Using snowballing sampling methods a further 10 people were suggested. All 29 people were contacted by email in first instance and those who did not respond by email were contacted by telephone. Of the 29 people approached, two declined to participate, seven did not respond to email or telephone contact, four agreed to participate but did not respond to further contact. Sixteen people provided written consent to participate in individual, semi-structured, in-depth interviews.

Participants were offered a telephone or face to face interview. Face to face interviews $(n=7)$ took place in the experts' place of work. All telephone interviews $(n=9)$ were conducted at the University of Manchester in a private office. All were audio recorded and transcribed verbatim. Table 1 shows the number of participants by professional background, level of academic expertise, area of expertise and training experience (some participants had more than one relevant area of expertise/training experience). 


\begin{tabular}{|c|c|}
\hline Professional background & Number of participants \\
\hline Health Psychology & 9 \\
\hline Public Health & 2 \\
\hline Nursing & 2 \\
\hline GP & 1 \\
\hline Social Work & 1 \\
\hline Psychiatry & 1 \\
\hline \multicolumn{2}{|l|}{ Academic expertise } \\
\hline Professor/Reader & 7 \\
\hline Senior Lecturer/Lecturer & 6 \\
\hline Research Fellow/Senior Researcher & 3 \\
\hline \multicolumn{2}{|l|}{ Area of academic expertise } \\
\hline Behaviour change (general) & 8 \\
\hline Healthcare professional behaviour change & 7 \\
\hline Mental Health/Psychiatry & 5 \\
\hline Implementation research & 3 \\
\hline Chronic illness/Self-management & 3 \\
\hline \multicolumn{2}{|l|}{ Training experience } \\
\hline Behaviour change skills for healthcare professionals & 7 \\
\hline Training in the context of research studies & 4 \\
\hline Motivational Interviewing skills for healthcare professionals & 4 \\
\hline Mental health management skills for healthcare professionals & 4 \\
\hline Patient self-management skills for healthcare professionals & 3 \\
\hline Communication skills for healthcare professionals & 3 \\
\hline Alcohol screening and brief intervention skills for healthcare professionals & 3 \\
\hline Changing healthcare professional behaviour & 2 \\
\hline
\end{tabular}


A topic based framework similar in content to the Study 1 matrix described above, but focused on healthcare professional training interventions in general, guided the overall content of the interviews.

\section{Analysis}

The analytical principles of detection, categorisation and classification (17) were used to guide the framework analysis. In Study 1, transcripts were indexed and summarised into the topic based framework using NVivo (first charting stage). Summarised data used participants own words as much as possible (18). Charted data within each theme was explored to look for overarching dimensions (detection), which were refined into fewer categories (categorisation) and examined for overarching, explanatory accounts (classification). In the classification stage, categories were examined together to develop an overarching explanatory account of what participants perceived to be effective training and why. The themes generated during this stage represented the assumed reasons for the perceived effectiveness or ineffectiveness of training components. Sub-themes within the themes emerged, and the data were reorganised and summarised into themes and sub-themes in an iterative processes which involved moving back and forth across the data until a coherent account (thematic framework) emerged (18).

In Study 2, the thematic framework developed in Study 1 was used to chart the data in NVivo (version 9.2). Following familiarisation of the interview transcripts, additional categories that did not map onto the sub-themes, but did map onto the higher order themes in the framework were identified. Data that could not be mapped onto the existing sub-themes were coded as 'other' within the theme. New sub-themes to account for the data were developed and further charting occurred. Participants' responses that mapped onto each theme were examined and organised into relevant training methods and implementation strategies. As higher order, explanatory themes formed the basis of the thematic framework developed in Study 1 , this analysis focused on describing responses that mapped onto the themes and identifying additional recommendations for effective training.

The data from both studies were interpreted using either the explicit responses of participants or underlying psychological theory (18). The main theory used to inform the interpretation process was Social Cognitive Theory (13-15). Where relevant, other psychological and learning theories were adopted in an inductive approach to assist interpretation and the development of a theoretical framework for understanding the perceived effectiveness of training intervention components.

The components identified as potentially effective during the analysis were defined and grouped to form a taxonomy of training intervention components. Each training component in the taxonomy was described using definitions or exemplars to enable understanding and a shared language to facilitate accurate reporting of interventions. Training components were grouped into clusters on the basis of their similarity in terms of what aspect of training they represented. Developing a taxonomy with a hierarchical structure involves organising similar intervention components into groups using both super- ordinate and sub-ordinate categories (20). The grouping labels were informed by guidance on the minimal detail required when reporting behaviour change interventions (content/elements, provider, format, setting, recipient, intensity, duration, fidelity) (10). Formal meetings among team members (KP, CB, LC) were used 
to agree labelling, definitions and exemplars. Revisions continued until there was an agreed set of intervention components and appropriate hierarchal groupings.

To test the comprehensiveness of the initial taxonomy, a systematic review of randomised controlled trials evaluating training interventions to improve the management of depression and anxiety in primary care (21) identified 38 studies, and the intervention descriptions were mapped to the proposed taxonomy. Any training intervention components identified that could not be easily mapped were added to the taxonomy within the appropriate groupings.

\section{Rigour}

In both studies, the appropriateness of the fit of the data mapped to the higher order themes was discussed during a series of meetings with the research team (KP, LC, CB) and any discrepancies were resolved. During this process, some of the themes and sub-themes were renamed to reflect the content, or grouped with other themes or sub-themes. The final version of the taxonomy was also agreed as part of an iterative process during a series of meetings with the team.

\section{Results}

Study 1 analysis identified five main themes (social interaction, credibility, relevance, information processing, and practicalities) of perceived effectiveness of training components, each of which contained several sub-themes. This culminated in the 'Perceived Effectiveness of Training' (PET) framework. Whilst developing an explanatory account of the data, it became clear that the themes represented three core concepts that need to be considered to optimise training for effectiveness: Interpersonal, Intrapersonal and System. Interpersonal relates to themes that involve other people, including the impact of other people on learning and social factors that influence effective training. Intrapersonal relates to any phenomenon concerned within the individual and incorporates how perceptions, beliefs/attitudes and the ability to process information are influenced by how training is delivered. System describes how training is evaluated in organisational or environmental terms, such as factors relating to the workplace or barriers and facilitators to implementation of the training objectives. These core concepts are akin to interaction between the person (intrapersonal), environment (interpersonal and system) and behaviour outlined in Social Cognitive Theory (SCT) (13), and can be viewed as separate but interrelated categories that influence each other.

The data analysed in Study 2 was found to map closely onto the PET framework. In addition it reflected the core concepts of interpersonal, intrapersonal and system factors as determinants of effective training. Only one sub-theme was not supported, which was 'mode of delivery' in the credibility theme. This possibly reflected the context of Study 1 being conducted as part of a training evaluation with some of the responses in interviews being specific to that training. Five new sub-themes emerged during the analysis of Study 2 which extended the PET framework. These included: Social support in 'Social Interaction'; Dual Processing in 'Information processing'; Training Practicalities in 'Practicalities'; Resources in 'Practicalities'; Reinforcement \& Incentives in 'Practicalities'. 
The final PET framework is presented in Fig. 1 below.

\section{Social Interaction}

The importance of social learning as a determinant of effective healthcare professional training was evident in both studies, and is widely acknowledge in the education field.

"I like the actual face to face learning, because it allows you to interact with colleagues, and actually, part of learning is actually being able to network and meet other people." (Study 1 , participant 6 )

Theories that can be used to explain the effectiveness of social interaction in healthcare professional training include SCT $(13,15)$, social identity theory $(22)$, Vygotsky's socio-cultural theory $(23)$ and the theory of planned behaviour (TPB) (24). Socialising and networking and learning from other people in training were strong sub-themes evident in both studies, thus can be seen as important aspects of social interaction that training should aim to encourage. The social influence sub-theme emerged from Study 1 in relation to concerns raised about diffusion of responsibility or social loafing (the reduced contribution to a group task by an individual) in group training. This sub-theme was expanded by the experts who evaluated training components in relation to whether they can instigate social influence and enable social comparison so that individuals are exposed to group norms. Designing a training programme that enables groups of healthcare professional to share examples of good practice may influence individuals' intentions; the theory of planned behaviour (24) identifies social norms as a predictor of intentions. This also highlights the need to manage negative normative influences on behaviour, as group processes in training may serve to reinforce negative attitudes or reasons not to change the behaviour.

Social anxiety can occur when individuals fear negative evaluation from others. Findings from both studies suggest that training that minimises anxiety and discomfort is perceived more positively by participants, but whether that leads to better learning outcomes remains to be tested empirically. Nonetheless, a challenge for facilitators is to ensure that mastery experiences are offered, as SCT would suggest that negative experiences of training caused by social anxiety could have detrimental effects on self-efficacy (15). Social support from peers and facilitators was identified by experts as important to learning and the implementation of training objectives in practice. Study 1 also identified the importance of training collaborating professionals together which could be one way to encourage social support amongst trainees.

\section{Credibility}

Perceived credibility of training as a determinant of effectiveness was another strong theme in both studies.

[ideal training programme] would be some sort of academic theories, again so linked to the actual course they're delivering, why they are actually delivering it, you know why they're being trained to deliver this course, selling the evidence... so selling the benefits to the organisation. (Study 2, participant 2) 
SCT $(13,15)$ (specifically the use of modelling techniques and persuasive sources to enhance selfefficacy) and key theories from the persuasion field such as source credibility (25) and the elaboration likelihood model (26) support this finding. The credibility of the facilitator warranted its own sub-theme because of the emphasis placed by participants in both studies on the importance of credible facilitators or trainers as a determinant of effective training, especially in relation to clinical experience and whether facilitators understand the realities of clinical practice. The inclusion of background evidence and/or theory to increase the credibility of the content of the training was also supported in both studies, showing that lecture based presentations that convey evidence and theoretical underpinnings of the new skill or behaviour being covered in training are essential to persuade healthcare professional to adopt a change in practice. Trainers need to acknowledge the difficulties healthcare professional face when trying to practice evidence based medicine in areas where there is uncertainty and present a convincing line of argument with relevant evidence to support whatever the training is trying to achieve. In addition to using credible facilitators and including background evidence to support the content of training, the mode of delivery or how and where training is delivered influenced perceptions of value or worth of a training programme in Study 1 , for example off-site training was seen to be more credible by some participants. This in turn could impact on behaviour change in practice so deserves consideration in the training design phase.

\section{Relevance}

Perceptions of relevance of the training were important determinants of the perceived effectiveness of the content and process of training in both studies.

"I think the role-play often falls down on the scenario being made up almost and not being kind of true. I think often it can be difficult to get a real sense of a scenario when it's made up... and it can become quite inconsistent as opposed to what it might be like if it was a real person with that real problem." (Study 1 , participant 1)

SCT (13) states that the capacity of individuals to reflect on past experiences and relate these to current thought processes facilitates understanding and learning. Participants in both studies discussed effective training in terms of the content being clear and relevant, which incorporates the use of suitable training methods and implementation strategies that are seen as relevant to the content of the training, the learning needs of trainees and their work in practice. The need for training to be grounded in reality was a major reason underlying perceptions of training as 'effective'. There was strong emphasis in both studies that training needs to reflect the reality of trainees' clinical practice and past experiences.

Preparation developed as a distinct sub-theme following emphasis of the importance of preparatory work in order for training to be effective. Experts particularly noted the importance of considering implementation strategies during the preparation stage so training can address possible barriers and facilitators to implementation. The result of preparatory work should lead to the training being perceived as more relevant, but may also result in more credible training. Structured training was also something that underpinned perceptions of effective training in both studies, and is linked to the need for trainees to 
have clear goals and objectives linked to their own personal learning objectives. This may also increase the relevance of the training with possible repercussions on perceived credibility.

\section{Information processing}

Training that incorporates strategies to facilitate information processing was a strong theme in participants evaluations of what makes training effective in both studies.

"I actually like more interactive really, because it makes you more alert, and you're less likely to let your mind wander off onto other things" (Study 1, participant 6)

Training methods that facilitate, consolidate or hinder the retention of information (memory) were discussed by participants in both studies, showing the need to consider working memory (27) in the design and delivery of training. The need to ensure the engagement of trainees during training was another strong subtheme in both studies and the skills of the facilitator are paramount here. The role of social interaction as a determinant of engagement in training was also clear. Absorbing information was another subtheme evident in both studies: specific training methods were seen to help the absorption of information to enhance learning (e.g. workshops with a mix of training components). The dual processing sub-theme identified in Study 2 is underpinned by dual processing theories $(26,28,29)$, which consider whether behaviour is automised/routinised or whether it requires more conscious effort to reenact. Experts discussed how this might be linked with behaviour change, and that training content should be considered in relation to the nature of the behaviour in question and whether it needs to become more habitual/routinised or whether habitual behaviour in practice is a barrier to achieving the aims of the training. Training should be focused on implementation and consider ways that might encourage the new behaviour(s) to become more habitual.

\section{Practicalities}

This theme relates to how training fits with the working practices of trainees, and environmental issues that might affect the quality of training. SCT $(13,15)$ supports this theme as a determinant of effective training, as environmental factors interact with the person to influence behaviour. Such factors include practical aspects of training and implementation that can act as barriers or facilitators,

"Say you have a practice nurse from a GP practice, and they come and they do the training and they are very enthusiastic and they go back into the workplace very motivated but unless that working environment, the structure of that working environment is amenable to accommodating that new training, delivering the result of that training, then it just dissipates and practice doesn't change" (Study 2, participant 1)

Participants in both studies discussed the need for training to be kept in-line with the working practices of trainees so that it causes minimal disruption and is seen as relevant to their work. There is some overlap with the relevance theme here, but these responses relate to more practical issues or system related issues that training should address. This theme was expanded quite significantly in Study 2, as practical 
issues relating to training were often mentioned by experts in relation to the perceived effectiveness of different aspects of training, which may reflect growing concerns with pragmatism and the need to address system barriers to behaviour change in the implementation field. This led to a new sub-theme, training practicalities, which encompasses practical matters important in relation to training itself, such as the benefits of on-line training when it needs to be delivered to large numbers. Having adequate resources to conduct training and implement training objectives was another practical need raised by experts in Study 2, who discussed how resources can influence the training methods and setting of the training and how this can affect the effectiveness of training. Finally, the reinforcement and incentives sub-theme describes experts' view that incentives can affect training attendance and behaviour change in practice, informed by substantial evidence from the behaviourist approach in psychology and the beneficial effects that positive reinforcement has on learning and behaviour (30). However, there were also some issues raised about the type of reinforcement that is most effective and the potential dangers or unintended consequences of using financial incentives to change behaviour, including creating expectations that it is only worth doing if they are paid to do it, thereby reducing intrinsic motivation.

\section{Links between themes}

The PET framework aims to show distinct factors underlying effective training, however themes are likely to interact. Themes that are especially related are social interaction and information processing (specifically engagement) and credibility and relevance, with perceptions of relevance likely to influence perceptions of credibility of the training. The core concepts (intrapersonal, interpersonal and system) can explain these interactions between themes; for example, social interaction in training can impact on an individual's learning and behaviour. It became clear during the analysis that all of the themes can be influenced by both the content and process (i.e. how it is delivered) of training. A key finding of this research was the centrality of the role played by the facilitator in delivering effective training. A highly skilled and knowledgeable facilitator can exert positive influences on all of the main themes in the PET framework and if necessary, their interjections can counteract a number of detrimental processes that would otherwise undermine training effectiveness.

\section{Training intervention component (TIC) taxonomy}

A range of training methods and implementation strategies were identified as having a positive impact on each theme/subtheme in the PET framework. These are presented in additional file 1, which can be used to identify training components that can address each theme in the framework.

The training intervention component (TIC) taxonomy is presented in supplementary file 2. The TIC taxonomy comprises a total of 171 training components (an overview of the taxonomy is shown in Box 1 below).

Box 1 - Overview of the TIC taxonomy showing the grouping labels and the number of training components identified in each group: 


\begin{tabular}{|lll|}
\hline Phase & Groupings & $\begin{array}{l}\text { Number of training } \\
\text { components }\end{array}$ \\
\hline Pre-training & Pre-training planning/preparation & 14 \\
\cline { 2 - 3 } $\begin{array}{l}\text { Training } \\
\text { delivery }\end{array}$ & Content & 3 \\
\cline { 2 - 3 } & Training methods & 7 \\
\hline & $\begin{array}{l}\text { Characteristics of the training } \\
\text { provider/facilitator }\end{array}$ & 82 \\
\hline & Characteristics of the recipients & 38 \\
\cline { 2 - 3 } & Length/duration & 6 \\
\cline { 2 - 3 } & Characteristics of the setting & 4 \\
\hline Post-training & Evaluation & 3 \\
\cline { 2 - 3 } & Skills transfer techniques & 5 \\
\cline { 2 - 3 } & Leadership & 6 \\
\hline
\end{tabular}

The training components in the 'content' group includes intervention strategies to inform the content (i.e. subject matter) of training as it is being delivered as opposed to behaviour change techniques or strategies which are described in other taxonomies (8). The term 'training methods' incorporates modes of delivery, exercises and activities and was used instead of 'format' to highlight the range of intervention components that can be used in the delivery of training. The term 'length/duration' reflects the duration (number of sessions) and intensity (contact time) as described by Davidson et al. (10).

\section{Discussion}

The PET framework was derived from the in-depth analysis of data from two qualitative studies of the views and experiences of independent healthcare professionals/experts. Five sub-themes were identified and organised around three core concepts; interpersonal, intrapersonal and system. The study findings suggest that effectiveness of training methods and implementation strategies in this area may be determined by how well the training fosters social interaction, perceived credibility and relevance, how it facilitates information processing and addresses practicalities (the real-life context of the training and working environment). If training facilitates interpersonal processes it may have a positive impact on individuals' knowledge, attitudes and behaviour. The design of training and the structure of the taught curricula should take account of intrapersonal factors such as how people learn and how their attitudes might influence behaviour. Training should also address system or organisational factors throughout the process (pre, during and post intervention). 
The identification of theoretical explanations for the perceived effectiveness of training supports any conclusions made about effective ways to train healthcare professionals and the PET framework identifies useful mechanisms and intervention targets. Recommendations about effective training methods and implementation strategies mapped against the framework (presented in additional file 1) can help training designers make an impact on the themes in the PET framework. Although there is a lack of previous research exploring effective healthcare professional intervention components, there have been some reviews in the area of improving the management of common mental health problems in primary care which was the context of the training the participants in study 1 received. Effective interventions were found to be multifaceted, conducted on-site, individually tailored and on-going (31). Another review reported no conclusive evidence for the effectiveness of any isolated educational methods, although there was some evidence that a combination of methods such as case studies, roleplay and feedback can improve diagnostic competency of GPs. They reported educational methods are often mentioned but not described, thus the effectiveness of effective educational methods is still unknown and requires further research (32). The TIC taxonomy could help to address this gap in the literature if used to describe training interventions and could help improve the quality of future healthcare professional training.

There are a number of ways to describe the different training components listed in additional file 1, which contains the same terminology as the TIC taxonomy. The taxonomy provides clear definitions to help to build a common language to describe training interventions. It is envisaged that it will be useful as an aid to design effective training, to improve the reporting of training interventions in published literature and to gather empirical evidence on the effectiveness of various components to training methods. The three phases of training (pre-training, training delivery and post training) in the taxonomy can aid intervention design and implementation processes and we recommend that the details of each phase should be reported in scientific reports for replication purposes. For example, describing pre-training planning/preparation components such as pre-training activities (exercises or activities that foster engagement and identify learning needs) is important information that would help others design effective training. Also, the 'incentives to attend training' group contain components that may be helpful to encourage people to attend training, and reporting whether or not these are effective would be highly informative and helpful to other researchers and help build an evidence base on the usefulness of incentives in this area.

The use of qualitative methodology facilitated the acquisition of in-depth understanding of what makes effective healthcare professional training and why particular aspects of training were perceived as effective or ineffective. For example, although many participants reported negative views about role-play, most understood the benefits of it and the value of participating in these exercises for learning. Therefore, the participants did not simply evaluate the training in terms of what they liked (which is commonly collected in training 'satisfaction' type evaluations) but meant that they considered training methods in terms of what is effective for their own learning. Focusing on the reasons for why something was perceived as effective/ineffective enabled a deeper understanding of why some training components were preferred, with the reasons interpreted using illuminative psychological theory. 
There is increasing recognition of the importance of involving patients and the public in research at the design stage and this should be an important part of training design. The identification of the target recipients of training and involvement of them in the design of training can help to ensure the training addresses their learning, as training needs analysis is an essential part of training development (3). In Study 1 , participants discussed how healthcare professionals want to have their needs assessed prior to training so the content is relevant to their learning needs. Furthermore experts can provide in-depth and efficient knowledge about a phenomenon (33) and because of difficulties identifying effective components of healthcare professional training in the literature, involving experts in training design is recommended. The inclusion of expert participants in study 2 lends strength and transferability to the findings of this research; and to the legitimacy of the PET framework in capturing what underpins effective training.

Ensuring rigour and transparency is a vital component of qualitative data analysis. The use of the Framework method to manage the data helped to ensure rigour in the data analysis of both studies. Framework matrices facilitate across and within case analysis to aid interpretation of the data, including identifying deviant cases or contradictions within cases and empty cells (34). Therefore, the use of the Framework method helped to ensure thorough and rigour analysis in both studies.

\section{Limitations}

Qualitative research does not allow examination of objective evidence of training effectiveness, such as the impact of healthcare professional training on patients. Quantitative research to test the predictive validity of the framework may be beneficial as a next step to gather further empirical evidence to support the conclusions about what constitutes effective training made in this research. Further scientific research on what makes healthcare professional training effective in different contexts is needed to support this research, and should aim to build a research agenda based on theory. The existing training literature comes from theoretically disparate disciplines (3), making it difficult to summarise and generalise to healthcare professional training. However, research on training in other fields outside of healthcare may be used to gather insights into what may influence effective healthcare professional training. Also, including a wider range of healthcare professionals may have identified further training components and the underlying reasons for their perceived effectiveness. Further research which employs purposive sampling strategies to take account of contextual differences is recommended.

The scope of this research was narrowed to focus on training interventions as a method of continued medical education to obtain a deeper understanding of this commonly used intervention to change healthcare professional behaviour in order to improve standards in the quality of care delivered to patients. It should also be noted that the training context in this research is quality improvement training concerned with evidence-based practice. Nonetheless it should be useful to anyone who designs or delivers healthcare professional training.

\section{Conclusion}


This research addresses some gaps in current knowledge about what constitutes effective training. The PET framework can be used for designing effective training to optimise the chances that it is positively received by healthcare professionals. Effective training is needed to translate evidence into practice and improve patient outcomes. The paper also provides recommendations about training content, training targets and methods of training delivery in relation to the themes and sub-themes which can be used as a guide to help training designers make an impact on the themes to maximise effectiveness. Future research can use the PET framework and TIC taxonomy to conduct theory based research on training effectiveness, including essential training evaluations. Testing the framework in other types of training would also widen its scope to other arenas thereby working towards a comprehensive framework of what constitutes effective healthcare professional training.

\section{Abbreviations}

CME

Continued Medical Education

PWP

Psychological Wellbeing Practitioners

PN

Practice nurses

PET

Perceived Effectiveness of Training framework

SCT

Social Cognitive Theory

TIC

Training Intervention Component taxonomy

\section{Declarations}

Ethics approval and consent to participate

Study 1: NHS ethical approval was obtained from Greater Manchester West Research Ethics Committee (reference 10/H1014/30). Study 2: Ethical approval for this study was obtained from the University of Manchester's Senate Committee on the Ethics of Research on Human Beings (reference 11078). Participants provided written consent in both studies.

Consent for publication

Not applicable

Availability of data and material 
The datasets used and/or analysed during the current study are available from the corresponding author on reasonable request.

Competing interests

The authors declare that they have no competing interests

Funding

This research was conducted as part of a PhD studentship funded by the Greater Manchester Collaboration for Leadership in Applied Health Research and Care (GM-CLAHRC). The funding body had no role in the design of the study and collection, analysis, and interpretation of data

Authors' contributions

KP undertook data collection and led the analysis and write up of the paper. LC and CB provided academic supervision for the work and contributed to data analysis and interpretation. All authors read and approved the final manuscript.

Acknowledgements

Not applicable

\section{References}

1. Chauhan BF, Jeyaraman M, Mann AS, Lys J, Skidmore B, Sibley KM, et al. Behavior change interventions and policies influencing primary healthcare professionals' practice-an overview of reviews. Implementation Science. 2017;12(1):3.

2. Michie S, Stralen MM, West R. The behaviour change wheel: a new method for characterising and designing behaviour change interventions. Implement Sci. 2011;6.

3. Salas E, Tannenbaum SI, Kraiger K, Smith-Jentsch KA. The Science of Training and Development in Organizations: What Matters in Practice. Psychological Science in the Public Interest. 2012;13(2):74101.

4. O'Brien MA, Rogers S, Jamtvedt G, Oxman AD, Odgaard-Jensen J, Kristoffersen DT, et al. Educational outreach visits: effects on professional practice and health care outcomes. Cochrane Database Syst Rev. 2007(4):CD000409.

5. Forsetlund L, Bjorndal A, Rashidian A, Jamtvedt G, O'Brien MA, Wolf F, et al. Continuing education meetings and workshops: effects on professional practice and health care outcomes. Cochrane Database Syst Rev. 2009(2):CD003030.

6. Oxman AD, Thomson MA, Davis DA, Haynes RB. No magic bullets: a systematic review of 102 trials of interventions to improve professional practice. CMAJ: Canadian Medical Association Journal. 1995;153(10):1423. 
7. Boutron I, Moher D, Altman DG, Schulz KF, Ravaud P. Extending the CONSORT statement to randomized trials of nonpharmacologic treatment: explanation and elaboration. Annals of Internal Medicine. 2008;148(4):295-309.

8. Michie S, Johnston M, Abraham C, Francis C, Eccles MP. The behavior change technique taxonomy (v1) of 93 hierarchically clustered techniques: Building an international consensus for the reporting of behavior change interventions. Annals of Behavioral Medicine. 2013;46(1):81-95.

9. Hoffmann TC, Glasziou PP, Boutron I, Milne R, Perera R, Moher D, et al. Better reporting of interventions: template for intervention description and replication (TIDieR) checklist and guide. BMJ : British Medical Journal. 2014;348.

10. Davidson KW, Goldstein M, Kaplan RM, Kaufmann PG, Knatterud GL, Orleans CT, et al. Evidencebased behavioral medicine: What is it and how do we achieve it? Annals of Behavioral Medicine. 2003;26(3):161-71.

11. EPOC. Data Collection checklist2002 6th September, 2013. Available from: http://epoc.cochrane.org/sites/epoc.cochrane.org/files/uploads/datacollectionchecklist.pdf.

12. Walter I, Nutley S, Davies H. Developing a taxonomy of interventions used to increase the impact of research2003 19th September, 2013. Available from: http://www.standrews.ac.uk/ ruruweb/pdf/Taxonomy\%20development\%20paper\%20070103.pdf.

13. Bandura A. Social foundations of thought and action. Englewood Cliffs, NJ: Prentice-Hall; 1986.

14. Bandura A. Self-efficacy: toward a unifying theory of behavioral change. Psychological review. 1977;84(2):191.

15. Bandura A. Self-efficacy: The exercise of control. New York: W.H Freeman and Company; 1997.

16. Johnson B, Christensen L. Educational Research: Quantitative, Qualitative, and Mixed Approaches, Research Edition, Second edition. Boston: Pearson Education Inc.; 2004.

17. Ritchie J, Spencer L. Qualitative data analysis for applied policy research. In: Bryman A, Burgess RG, editors. Analyzing qualitative data. London: Routledge; 1994. p. 173-94.

18. Ritchie J, Spencer L, O'Connor W. Carrying out qualitative analysis In: Ritchie J, Lewis J, editors. Qualitative research practice: A guide for social science students and researchers. London: Sage; 2003. p. 219-62.

19. Coventry PA, Lovell K, Dickens C, Bower P, Chew-Graham C, Cherrington A, et al. Collaborative Interventions for Circulation and Depression (COINCIDE): study protocol for a cluster randomized controlled trial of collaborative care for depression in people with diabetes and/or coronary heart disease. Trials. 2012;13:139.

20. Stavri Z, Michie S. Classification systems in behavioural science: current systems and lessons from the natural, medical and social sciences. Health Psychology Revew. 2012;6(1):133-40.

21. Perryman K. Sythesising existing and developing new evidence on effective healthcare professional training that aims to improve hte management of psychological distress in primary care: Univeristy of Manchester; 2014. 
22. Tajfel H, Turner JC. An integrative theory of intergroup conflict. The social psychology of intergroup relations. 1979;33:47.

23. Vygotsky LS. Mind in society: The development of higher psychological processes. Cambridge, MA: Harvard University Press; 1978.

24. Ajzen I. The theory of planned behavior. Organizational behavior and human decision processes. 1991;50(2):179-211.

25. Hovland $\mathrm{Cl}$, Weiss $\mathrm{W}$. The influence of source credibility on communication effectiveness. Public opinion quarterly. 1951;15(4):635-50.

26. Petty RE, Cacioppo JT. The elaboration likelihood model of persuasion. In: Berkowitz L, editor. Advances in experimental social psychology. 19. New York: Academic; 1986. p. 123-205.

27. Baddeley A. Working memory. Science. 1992;255(5044):556-9.

28. Chen S, Chaiken S. The heuristic-systematic model in its broader context. In: Chaiken S, Trope Y, editors. Dual-process theories in social and cognitive psychology. New York: Guildford; 1999. p. 7396.

29. Strack F, Deutsch R. Reflective and Impulsive Determinants of Social Behaviour. Personality and Social Psychology Review. 2004;8(3):220-47.

30. Skinner BF. Science and human behavior. New York: MacMillan; 1953.

31. Kroenke K, Taylor-Vaisey A, Dietrich AJ, Oxman TE. Interventions to improve provider diagnosis and treatment of mental disorders in primary care: A critical review of the literature. Psychosomatics. 2000;41(1):39-52.

32. Benthem GHH, Heg RR, van Leeuwen YD, Metsemakers JFM. Teaching psychiatric diagnostics to general practitioners: Educational methods and their perceived efficacy. Medical Teacher. 2009;31(7):e279-86.

33. Bogner A, Littig B, Menz W, editors. Interviewing experts. Bastingstoke, UK: Palgrave Macmillan; 2009.

34. Gale NK, Heath G, Cameron E, Rashid S, Redwood S. Using the framework method for the analysis of qualitative data in multi-disciplinary health research. BMC Medical Research Methodology. 2013;13(1):117.

\section{Additional Files}

Additional file 1.docx. Recommended training components identified in each - this file contains a table of training intervention components identified as having a positive impact on each theme/subtheme in the PET framework

Additional file 2.docx. The Training Intervention Component (TIC) taxonomy showing evidence source (ES) - this file contains the full taxonomy, showing source of evidence.

\section{Figures}




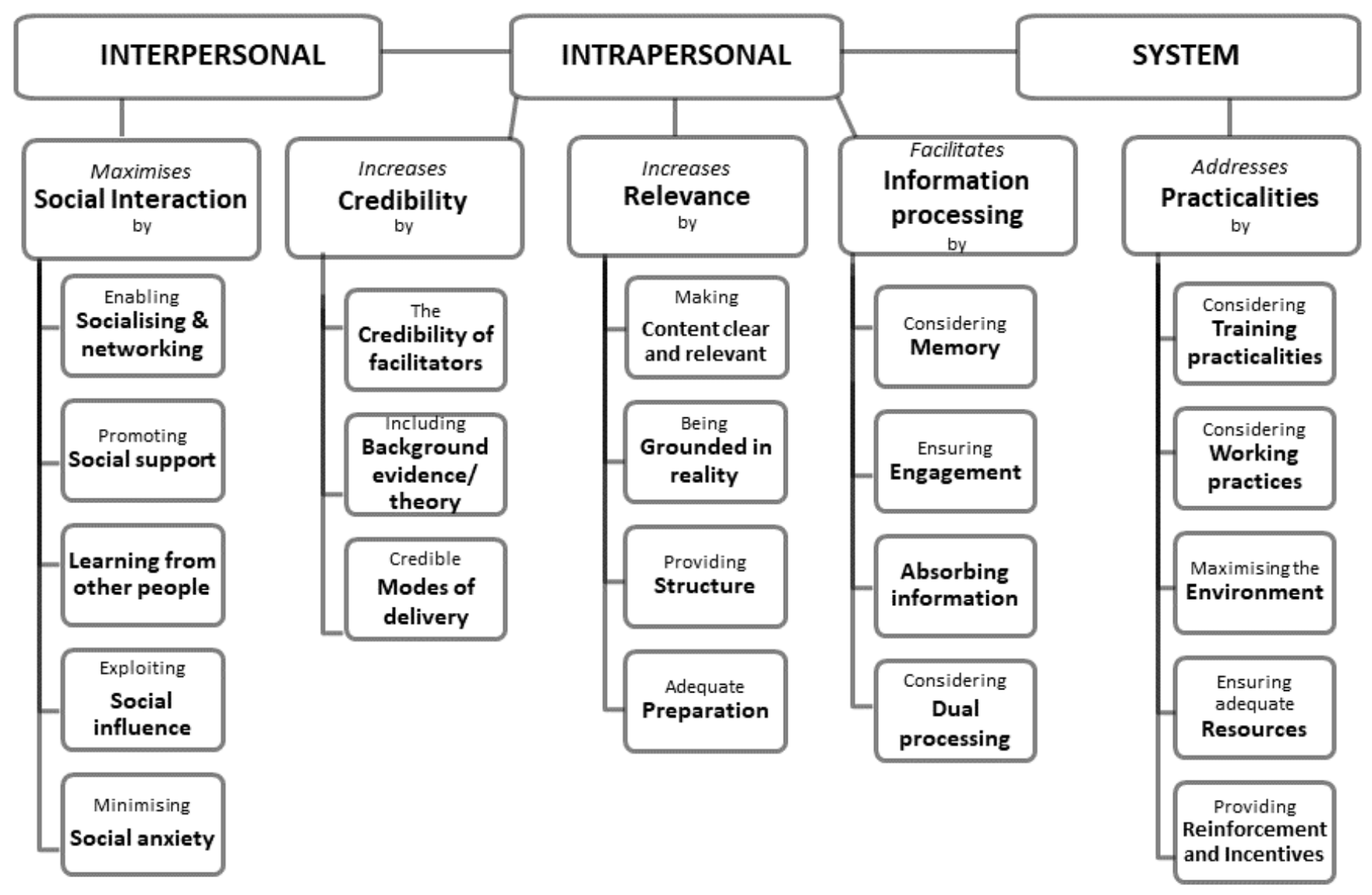

Figure 1

The Perceived Effective of Training Framework

\section{Supplementary Files}

This is a list of supplementary files associated with this preprint. Click to download.

- Additionalfile1.docx

- Additionalfile2.docx 